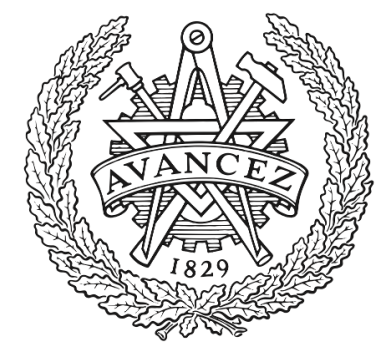

CHALMERS

UNIVERSITY OF TECHNOLOGY

\title{
A Review on Challenges and Solutions in Microgrid Protection
}

Downloaded from: https://research.chalmers.se, 2023-04-26 09:42 UTC

Citation for the original published paper (version of record):

Srivastava, A., Mohanty, R., Fotouhi, A. et al (2021). A Review on Challenges and Solutions in Microgrid Protection. 2021 IEEE Madrid PowerTech, PowerTech 2021 - Conference Proceedings. http://dx.doi.org/10.1109/PowerTech46648.2021.9495090

N.B. When citing this work, cite the original published paper. 


\title{
A Review on Challenges and Solutions in Microgrid Protection
}

\author{
Ankur Srivastava, Rabindra Mohanty, M. Ali Fotouhi Ghazvini, Le Anh Tuan, David Steen, Ola Carlson \\ Division of Electric Power Engineering \\ Department of Electrical Engineering \\ Chalmers University of Technology \\ 41296 Gothenburg, Sweden \\ Email: \{ankur.srivastava, rabindra, ali.fotouhi, tuan.le, david.steen, ola.carlson\}@ chalmers.se
}

\begin{abstract}
Protection of microgrid has become challenging due to the hosting of various actors such as distributed generation, energy storage systems, information and communication technologies, etc. The main protection challenges in the microgrid are the bi-directional power flow, protection blinding, sympathetic tripping, change in short-circuit level due to different modes of operation, and limited fault current contribution by converterinterfaced sources. This paper presents a comprehensive review of the available microgrid protection schemes which are based on traditional protection principles and emerging techniques such as machine learning, data-mining, wavelet transform, etc. A categorical assessment of the reviewed protection schemes is also presented. The key findings of the paper suggest that the timedomain and communication-assisted protection schemes could be suitable solutions to address the identified protection challenges in the microgrid.
\end{abstract}

Index Terms-Distributed generation, distribution systems protection, microgrid, microgrid protection.

\section{INTRODUCTION}

Climate change concerns are leading the transition in power generation from conventional fossil fuels to renewable energy sources (RES). This transition has brought a change in the protection methods for the distribution system operators. The concept of microgrids (MGs) has added further complexity to the protection schemes. The condition with excessive RES generation and light or no-load conditions could force the system operators to shut down the power generation due to voltage variation issues and network congestion problems; a few of such incidents are reported in [1], [2].

MG may have bidirectional power flow, wide operational range, etc. and can operate as a controlled entity both in grid-connected and islanded modes. MG can be classified into radial and ring type networks. From protection point of view, the power flow in radial network is unidirectional while in case of ring network it could be bidirectional. In case of radial systems with distributed generation (DG) interconnection in downstream, the power flow could also be bidirectional. Thus, the MG protection schemes developed for ring networks are also suitable for radial networks. The main challenges which drive the need to revisit the conventional protection schemes include the following:

The work presented in this paper is financially supported by the following projects: i) UNITED-GRID - received funding from the European Community's Horizon 2020 Framework Programme under grant agreement no. 773717; ii) FLEXI-GRID - received funding from the European Community's Horizon 2020 Framework Programme under grant agreement no. 864048; iii) WP-A6 - received funding from Energy Area of Advance of Chalmers University of Technology, Sweden.
1) High penetration of RES may lead to reverse power flow or bi-directional power flow, which causes relay coordination problems and failures in the conventional protection schemes with no directional element [3]-[5].

2) Changes in the mode of operation of MGs from gridconnected to islanded mode and vice-versa could have a substantial impact on the short-circuit level due to change in equivalent impedance [6], [7].

3) Fault current limitations by the converter-based DGs could lead to undetected fault. voltage and frequency problems during islanding, etc. [8], [9].

There is ongoing research to address these protection challenges in MG. Some researchers have proposed new methods that are based on traditional protection principles such as adaptive overcurrent, directional overcurrent, undervoltage, fault loop impedance, and sequence component. Simultaneously, in the era of the evolution of new techniques such as machine learning (ML), data-mining, Internet of things, synchrophasors, multi-agents, etc., researchers are also utilizing these promising techniques in addressing the protection challenges in MG. Some research works in [8]-[11] have presented a review of the protection schemes in MG. However, still, a categorical review of $\mathrm{MG}$ protection schemes based on traditional protection principles and the special techniques is missing.

To address the aforementioned gap, this paper presents a categorical review of various traditional protection principles based schemes proposed for MG. Also, a comprehensive review of protection schemes that applies special techniques in addressing the challenges, is presented in the paper. A comparative assessment with general advantages, area of improvements and potential applications for the different protection categories is also presented to summarize the review work.

The rest of the paper is organised as follows. Section II discusses the MG protection schemes based on traditional protection principles. The special techniques based protection schemes are reviewed in Section III. The discussions and concluding remarks are presented in Section IV.

\section{MG PROTECTION SCHEMES BASED ON TRADITIONAL PROTECTION PRINCIPLES}

This section reviews the MG protection schemes for both grid-connected and islanded modes of operation, which are developed based on the traditional protection principles. Due 
to the protection challenges as discussed in Section I, the conventional protection schemes are required to be re-designed for MG. The relay protection settings would be different and depends on the operating mode of MG. There are also adaptive protection schemes which are suitable for both gridconnected and islanded operating modes. These protection challenges in a $\mathrm{MG}$ are addressed through the following protection schemes. The schematics of a grid-connected MG with different components is presented in Fig. 1.

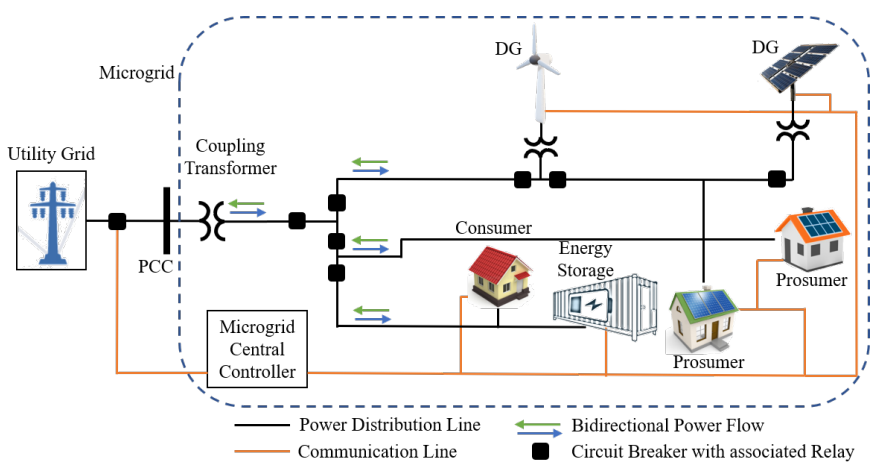

Fig. 1. Schematics of intelligent microgrid with different components.

\section{A. Protection Schemes for Grid-connected Microgrid}

1) Current-based Protection Schemes: Current-based protection schemes includes overcurrent, differential, current-only directional principles. An adaptive phase current-based protection method is proposed in [3]. The effect of high penetration of DGs on relay coordination is explored first. Subsequently, fault detection is performed using the current contribution information from all the sources, including the main grid. The faulted section is identified by the pre-calculation of Thevenin equivalent impedance of each source for different fault types.

A protection strategy is presented in [12] where the MG is not disconnected from the main grid in case of a MG fault. The overcurrent relay performs correctly and selectively clears the fault due to the large fault current supplied by the grid. For a fault on the grid side, the MG gets disconnected and operates in an islanded mode. In case, if a fault occurs within the MG in islanded mode, it is considered as a second fault, resulting in the disconnection of the MG DGs (after a certain time delay) to avoid any undesirable situation.

In grid-connected mode, directional overcurrent relay distinguishes the forward and reverse direction faults. For proper coordination, a definite time delay is added in the reverse mode. The negative sequence component of current is incorporated in the directional unit to detect unbalanced faults in MG [13].

2) Voltage-based Protection Schemes: The three-phase balanced faults do not contain negative and zero-sequence components and thus an undervoltage relay accompanied with a directional element can be used for the protection during the balanced fault [6]. Also, a definite time delay is utilized for coordinated operation in forward and reverse direction of fault [13].

3) Both Voltage and Current based Protection Schemes: Protection principles based on impedance, reactance, and the directional unit use both voltage and current for the protection decision. Usually, impedance based protection schemes are deployed for transmission level, but being free from fault calculations, they also find applications in MG protection. An impedance-based protection scheme for MG is discussed in [7]. However, it's performance in a system with multiple tapped feeders is not reliable due to current in-feed.

\section{B. Protection Schemes for Grid-disconnected (Islanded) $\mathrm{Mi}$ - crogrid}

The subsection discusses the protection schemes where the MG is islanded from the main grid due to any reason.

A comprehensive digital protection scheme is proposed in [14] for MGs operating in both grid-connected and islanded modes. The current differential technique is used for line protection. While, overcurrent, undervoltage, negative sequencebased protection functions are used for DG and point of common coupling (PCC) protection.

A dynamic adaptive overcurrent relay is presented in [15] for the protection of MG in islanded mode. The relay changes the pickup current settings by using the DG status.

A symmetrical component-based protection scheme is proposed in [16], where the fault is detected by obtaining the positive-sequence impedance of the line. Thereafter, the correct faulted section is identified by comparing the upstream and downstream positive sequence impedance during fault and pre-fault conditions.

An integrated impedance angle (computed from positive sequence voltage and current phasors) based protection scheme is presented in [17] for MGs in islanded operation. An internal fault is confirmed when the angle is in the range of $-5^{\circ}$ to $-90^{\circ}$; otherwise, it is treated as an external fault.

The rate of change of voltage phase angle of a bus with respect to PCC is used for fault detection in [18]. The method uses a phasor measurement unit (PMU) with IEEE C37.118.1 standard for angle estimation and the rate of angle difference between two buses is an indicator of fault identification.

The sum of the phase and neutral currents are used in [19] to detect the fault in MG, where the entire MG is divided into several protection zones, and a circuit breaker is connected between the zones to isolate the minimum part during a fault.

\section{Islanded (Stand-alone) Microgrid Protection Schemes}

This subsection discusses the protection schemes when the MG operates in islanded mode only.

A protection scheme based on inverter-control and microprocessor-relay (MR) settings is proposed in [20]. The scheme injects a percentage of the fifth harmonic into the fault current using software current control to support MR in accurately identifying the fault. The inverter is also protected against short-circuit faults by limiting currents using hardware hysteresis and software current control.

An adaptive overcurrent protection scheme is proposed in [21] utilizing programmable microprocessor-based intelligent electronic devices. These devices communicate among themselves and with a central controller to make a decision.

An active protection method based on differential protection and injection of off-nominal frequency during fault conditions through the inverter-based DG control is proposed in [22] for islanded MG. 
A new time-domain model for inverter-interfaced DG is proposed to investigate the protection issues in islanded $\mathrm{MG}$ [23]. The protection issues in islanded MG completely fed by DG or have very high DG penetration are addressed in [24].

\section{Anti-Islanding Protection Schemes}

Unintentional islanding of a $\mathrm{MG}$ is a concern as it causes safety hazards for personnel and damaging of equipment due to out-of-phase tripping [25]. The anti-islanding requirement in DG is adopted to avoid such severe issues and hence the DG must be disconnected from the network followed by islanding.

The anti-islanding protection is broadly classified into three categories as active, passive, and hybrid detection strategies [26]. Active strategies amplify the small perturbation generated in frequency, phase angle, and harmonics during the disturbance. Passive strategies are based on under/overvoltage and under/over frequency at the PCC. Hybrid strategies are based on the exploitation of the strength of both passive and active strategies where passive detection is used first. Thereafter, perturbations are injected for enabling the active methods.

\section{Special Techniques Based Protection Schemes}

This section reviews the protection schemes which employs special techniques in protection design for $\mathrm{MG}$ operating in both grid-connected and islanded modes.

\section{A. Setting-less Protection Schemes}

Setting-less protection is a novel approach based on dynamic state estimation. The proposed method has the potential to address the existing protection gaps and also improving zone protection [27].

The concept of setting-less protection also referred to as dynamic state estimation based protection (DSEBP), is derived from the differential protection and can be regarded as it's generalization. DSEBP utilizes the voltage and current measurements from inside and the terminals of the protection zone, speed and torque measurements in case of rotating devices, or other internal measurements (such as thermal measurements).

DSEBP does the dynamic modelling of physical laws for protection zone such as Kirchhoff's current and voltage, thermodynamics, and motion law's which should be obeyed by all the measurements. Thus, in the absence of any internal fault in the protection zone, all the physical laws should be satisfied by the measurements while vice-versa in case of any internal fault, leading to a clear, secure, and reliable identification of the internal faults [27].

DSEBP can also be applied for $\mathrm{MG}$ protection to address the various challenges. A DSEBP scheme is proposed in [28] for all the MG components. The work demonstrates the application and advantages of this scheme for MG circuits by comparing the performance with the traditional protection techniques. A distributed dynamic state estimation is used to achieve adaptive grid protection in [29]. Further, DSEBP is used to design a centralized protection scheme for MG in [4].

\section{B. Synchrophasor Assisted Protection Schemes}

The integrated impedance angle based protection scheme is proposed in [17] which uses PMU retrieved positive sequence voltage and currents from both ends of the lines to detect the fault type, location, and resistance. The scheme is robust in distinguishing the critical issues from the faults, and thus it could be a potential candidate for developing centralized protection schemes for MG.

An effective, fast, and reliable fault detection scheme is proposed in [18] for different operating conditions of MG. The method detects the faults by monitoring the absolute value of the rate of angle difference of the phase voltage between the PCC and the bus closest to the faulted line by using PMUs.

A MG protection scheme based on the positive-sequence component with the capability of single-phase tripping is developed in [16]. It employs PMUs and microprocessorrelay based on a digital communication platform to enable the protection of MGs in looped and radial configurations under both operating modes.

\section{Travelling Wave based Protection Schemes}

The single-phase-to-ground fault is detected by a novel travelling wave-based protection scheme proposed in [30]. The method determines the fault direction by comparing the polarities of current and voltage travelling waves, which are measured immediately when the fault occurs.

The initial current travelling wave-based protection scheme is introduced in [31] with specific logic for meshed networks and feeders with single-end measurement. The proposed scheme is based on the mathematical morphology technology and a simplified polarity detection method.

A travelling wave theory-based protection algorithm for medium voltage lines is presented in [32]. The algorithm determines the fault direction by analyzing the differential equations, which describes the behavior of fault-induced current waves. The neural network-based classifiers are used to distinguish between the internal and external faults. This approach only requires local measurements of current. Information exchange is not required as frequently as compared to other protection schemes, and thus it could be implemented using less communication links. These characteristics make it a reliable and low-cost solution for the protection of the MGs. The shortcomings of [32] are resolved in [33] by using both voltage and current measurements. The main advantage is the use of physical considerations compared to the data-driven ML algorithm previously used.

\section{Machine Learning based Protection Schemes}

A reliable adaptive protection scheme based on ML techniques is presented in [34]. The scheme first uses Pearson correlation coefficients from data-mining to analyze uncertain elements and then a hybrid artificial neural network and support vector machine (SVM) model for MG state recognition based on big data streams. Finally, the proposed scheme determines the adaptive protection settings and network reconfigurations.

A new method based on a semi-supervised ML technique with the co-training of decision tree and k-nearest neighbour is proposed in [35] for the fault classification in MG. It provides better performance as compared to previous supervised ML techniques due to the use of both labeled and unlabeled data for building and training of the classifier model. 
A MG protection scheme proposed in [36] is based on the Hilbert-Huang transform (HHT) and ML techniques. The scheme initially extracts the differential features from specific current signals using HHT, which are then fed as input to the three different ML models, i.e., Naive Bayes classifier, SVM, and extreme learning machine, for the detection and classification of MG faults.

A protection scheme based on a sparse auto encoder and deep neural network is proposed in [37] to clearly distinguish array and symmetrical line faults for PV-integrated MG. The proposed scheme has added features like fault detection and classification, section identification, and mode detection.

ML algorithms based adaptive protection scheme for $\mathrm{MG}$ in both grid-connected and islanded modes is proposed in [38]. The work shows that relay measurements could be used to obtain detection features for ML-based prediction models to detect the faults accurately.

A supervised ML method using principal component analysis to extract features is proposed in [39] for fault detection.

A MG protection scheme based on wavelet transform (WT) and deep neural networks is proposed in [40]. The scheme samples and prepossesses the current signals using discrete WT for the extraction of statistical features. The fault detection is done by giving these features as inputs to deep neural networks for training and testing.

\section{E. Wavelet Transform based Protection Schemes}

A combined WT and decision tree-based MG protection scheme is proposed in [41]. The effective features such as deviation in energy and entropy, etc., are calculated by obtaining and prepossessing the current signals through WT. After the feature extraction, training and validation of the decision tree is performed. Finally, the fault classification is done by the inclusion of both wavelet-based and current signals features.

A protection scheme based on WT and ensemble of the SVM classifier is proposed in [42]. It provides the standard deviation of coefficients obtained by the feature extraction process of WT as inputs ensemble of SVM for training.

An adaptive distance protection scheme is proposed in [43] for inverter-based DG composed MG based on complex WT. The scheme generates the relay command and setting by coordination optimization model using an ant colony algorithm.

\section{F. Multi-agents based Protection Schemes}

A multi-agents based differential protection scheme with variable tripping time is proposed in [44]. The agents have their primary, backup, and bus protection layers, which are used for primary and backup protection.

A protection scheme based on Q-learning algorithm and multi-agent systems is proposed in [45]. The agents are trained using the Q-learning algorithm for fault identification and clearance. Further, the agents communicate with each other using the decentralized blockchain-based connections.

A protection scheme is proposed in [46] using resilient intelligent agents. The voltage and current measurements are used for fault detection. The components act as agents and communicate the decision to upper control levels and neighbouring components for performance enhancement.
A multi-agents based MG protection scheme is proposed in [47] where the MG components like DGs, relays, PCC, controllers act as agents. The scheme uses the adaptive protection where the pre-contingency decentralized adaptive protection interacts with the MG central controller to adapt the new protection settings in post-contingency conditions.

A MG protection scheme for fault detection, clearing, and localization, along with the restoration method based on a communication-assisted multi-agents system, is proposed in [48]. The agents are present at the end and middle of each section and compare the phase angles of current signals from both sides to produce the relay decision.

A scheme using a co-simulation platform based on a multiagent system is proposed in [49]. It utilizes a supercapacitor bank for the resiliency enhancement and does not require different settings with the change in MG configuration.

\section{G. Data-Mining based Protection Schemes}

A data-mining patterns and classification algorithms based fault detection method in MG is proposed in [50]. Two different classifiers, i.e., random forest and K-nearest neighbors, are used to distinguish the fault features with no-fault conditions which are obtained by wavelet packet transform.

A comprehensive data-mining based differential protection scheme is proposed in [51]. The voltage and current signals during the fault are prepossessed to estimate the sensitive features. After that, the differential features are computed from these features which are further used to build a decision treebased data-mining model to obtain the final relay output.

\section{Discussions And Concluding Remarks}

From the protection challenges as discussed in Section I and review of protection schemes for MGs, the following can be summarized.

- The challenge associated with the bi-directional power flow including the sympathetic tripping and protection blinding is addressed in [4], [5], [7], [10]-[14], [17]-[19], [24], [25], [28], [33], [37].

- The challenge associated with the change in the mode of operation is addressed in [4], [7], [10], [11], [13]-[21], [23], [25], [26], [28], [31], [33]-[38], [40], [41], [44], [49]-[51].

- The challenge associated with the fault current limitation is addressed in [4], [7], [13], [14], [16], [19]-[26], [43].

A summary along with the comparative assessment including the general advantages, area of improvements, and potential applications associated with different categories of the protection schemes, is presented in Table I. The following inference could be made from the comparative assessment that the time-domain and communication-assisted protection schemes could be the feasible solutions for achieving fast relay operation in future low-inertia MGs. However, in the case of time-domain schemes, issues such as low signal strength of travelling wave for a certain fault inception instant and switch-on-to-fault (unavailability of pre-fault data) in superimposed component, while, communication failure and cyberattack in case of communication-assisted schemes, could be possible areas of developments. 
TABLE I

A Summary of Microgrid Protection Schemes

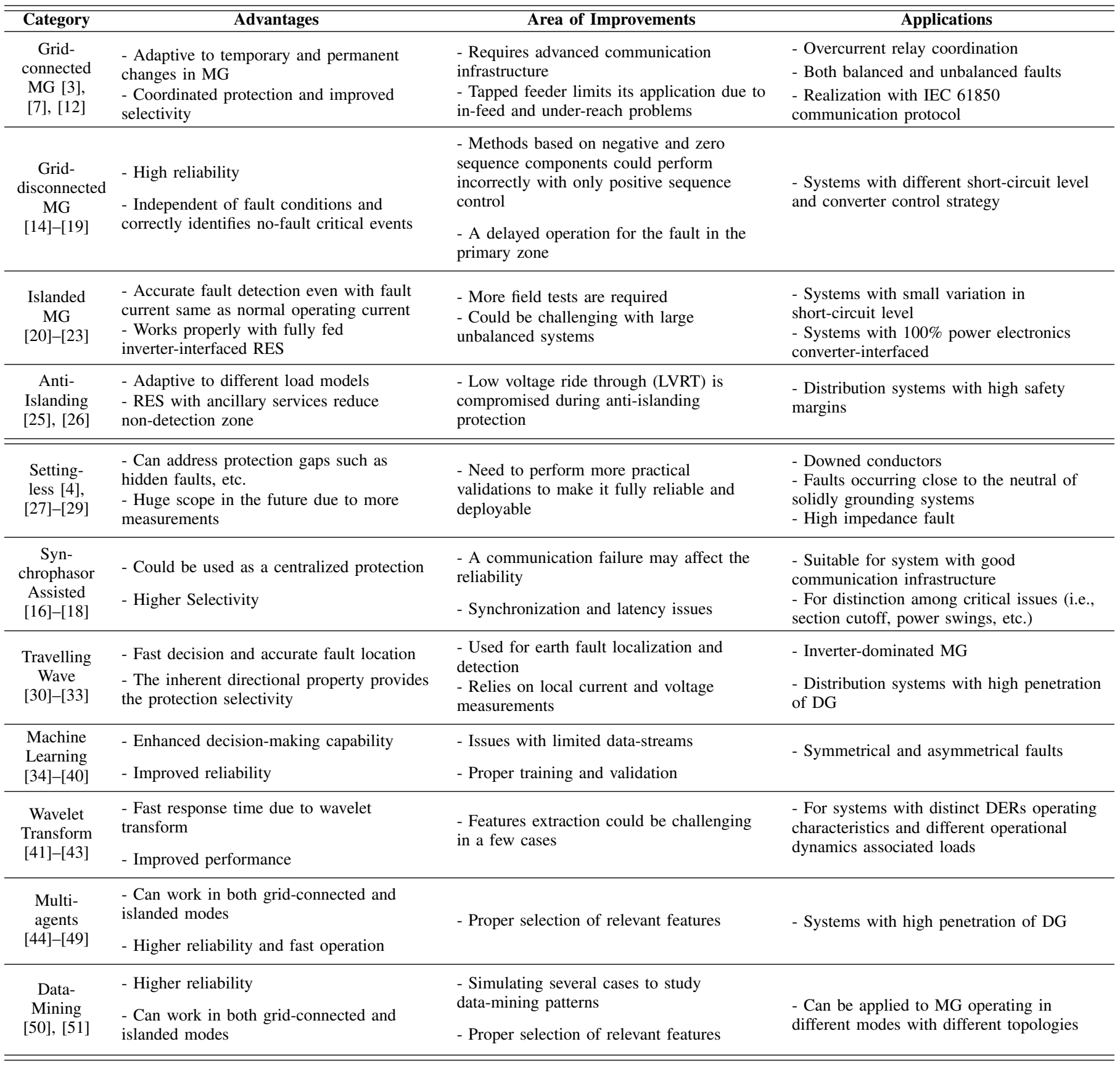

Recently, the protection of MG has become a challenge due to the changing power generation scenario from fossilbased to RES. This review paper identifies the main challenges in the MG protection as bi-directional power flow causing coordination problems, impact on short-circuit level due to change in the mode of operation, and fault current limitations by converter-interfaced DGs. Thereafter, the paper classifies the presently available protection schemes into two different categories. The research gaps identified from the review can be summarized. i) how can cost-efficient communication-assisted protection be designed which is robust to communication delay and link failures, ii) how can the field and practical validations be enhanced for special techniques based protection schemes, and iii) how can the data and latency issues be handled for synchrophasor based protection schemes. The parameters such as speed, sensitivity, and reliability are important to be considered in designing a protection scheme for future lowinertia MGs. This review paper will support the researchers working in the field of MG protection, to get an overview of ongoing research and current state-of-the-art.

\section{REFERENCES}

[1] Liz Hobday, "Electricity distributors warn excess solar power in network could cause blackouts, damage infrastructure," Australian Broadcasting Corporation, Oct. 2018.

[2] Giles Parkinson, "Utilities move to kick rooftop solar off the grid," Renew Economy, June 2014. 
[3] S. M. Brahma et al., "Development of adaptive protection scheme for distribution systems with high penetration of distributed generation," IEEE Trans. Power Delivery, vol. 19, no. 1, pp. 56-63, Jan 2004.

[4] O. Vasios, S. Kampezidou, and A. P. S. Meliopoulos, "A dynamic state estimation based centralized scheme for microgrid protection," in Proc. IEEE North American Power Symposium (NAPS), 2018, pp. 1-6.

[5] V. A. Papaspiliotopoulos et al., "Hardware-in-the-loop design and optimal setting of adaptive protection schemes for distribution systems with distributed generation," IEEE Transactions on Power Delivery, vol. 32, no. 1, pp. 393-400, Feb 2017.

[6] V. Telukunta et al., "Protection challenges under bulk penetration of renewable energy resources in power systems: A review," CSEE Journal of Power and Energy Systems, vol. 3, no. 4, pp. 365-379, 2017.

[7] S. M. Brahma et al., "Insight into microgrid protection," in Proc. IEEE PES Innovative Smart Grid Technologies Europe, 2014, pp. 1-6.

[8] P. Naveen and P. Jena, "A review on issues and coordination strategies for over current protection in microgrid," in Proc. 14th IEEE India Council International Conference (INDICON), 2017, pp. 1-6.

[9] A. Khademlahashy et al., "A review on protection issues in micro-grids embedded with distribution generations," in Proc. 12th IEEE Conference on Industrial Electronics and Applications (ICIEA), 2017, pp. 913-918.

[10] J. Shiles et al., "Microgrid protection: An overview of protection strategies in North American microgrid projects," in Proc. IEEE Power \& Energy Society General Meeting (PESGM), 2017, pp. 1-5.

[11] X. Kang, C. E. Nuworklo, B. S. Tekpeti, and M. Kheshti, "Protection of micro-grid systems: a comprehensive survey," The Journal of Engineering, vol. 2017, no. 13, pp. 1515-1518, 2017.

[12] B. Hadzi-Kostova, Z. Styczynski, and R. Krebs, "New protection concepts for distribution systems with dispersed generation," in Proc. IEEE Russia PowerTech, 2005, pp. 1-6.

[13] A. Hooshyar and R. Iravani, "Microgrid protection," Proceedings of the IEEE, vol. 105, no. 7, pp. 1332-1353, 2017.

[14] S. F. Zarei and M. Parniani, "A comprehensive digital protection scheme for low-voltage microgrids with inverter-based and conventional distributed generations," IEEE Transactions on Power Delivery, vol. 32, no. 1, pp. 441-452, Feb 2017.

[15] R. Jain, D. L. Lubkeman, and S. M. Lukic, "Dynamic adaptive protection for distribution systems in grid-connected and islanded modes," IEEE Transactions on Power Delivery, vol. 34, no. 1, pp. 281-289, Feb 2019.

[16] S. Mirsaeidi et al., "A protection strategy for micro-grids based on positive-sequence component," IET Renewable Power Generation, vol. 9, no. 6, pp. 600-609, 2015.

[17] N. K. Sharma and S. R. Samantaray, "PMU assisted integrated impedance angle based microgrid protection scheme," IEEE Transactions on Power Delivery, vol. 35, no. 1, pp. 183-193, Feb 2020.

[18] N. K. Sharma and S. R. Samantaray, "Assessment of PMU-based widearea angle criterion for fault detection in microgrid," IET Generation, Transmission \& Distribution, vol. 13, no. 19, pp. 4301-4310, 2019.

[19] H. Nikkhajoei and R. H. Lasseter, "Microgrid protection," in Proc. IEEE Power Engineering Society General Meeting (PESGM), 2007, pp. 1-6.

[20] Z. Chen et al., "A novel protection scheme for inverter-interfaced microgrid (IIM) operated in islanded mode," IEEE Transactions on Power Electronics, vol. 33, no. 9, pp. 7684-7697, 2017.

[21] K. Lai, M. S. Illindala, and M. A. Haj-Ahmed, "Comprehensive protection strategy for an islanded microgrid using intelligent relays," in Proc. IEEE Industry Applications Society Annual Meeting, 2015, pp. 1-11.

[22] A. Soleimanisardoo, H. K. Karegar, and H. H. Zeineldin, "Differential frequency protection scheme based on off-nominal frequency injections for inverter-based islanded microgrids," IEEE Transactions on Smart Grid, vol. 10, no. 2, pp. 2107-2114, 2018.

[23] S. Brahma, N. Pragallapati, and M. Nagpal, "Protection of islanded microgrid fed by inverters," in Proc. IEEE Power \& Energy Society General Meeting (PESGM), 2018, pp. 1-5.

[24] S. Brahma, "Protection of distribution system islands fed by inverterinterfaced sources," in Proc. IEEE Milan PowerTech, 2019, pp. 1-6.

[25] F. Blaabjerg et al., "Distributed power-generation systems and protection," Proceedings of the IEEE, vol. 105, no. 7, pp. 1311-1331, 2017.

[26] D. Pal and B. K. Panigrahi, "Analysis and mitigation of the impact of ancillary services on anti-islanding protection of distributed generators," IEEE Transactions on Sustainable Energy, pp. 1-1, 2020.

[27] A. P. S. Meliopoulos et al., "Dynamic state estimation-based protection: Status and promise," IEEE Transactions on Power Delivery, vol. 32, no. 1, pp. 320-330, 2016.

[28] Y. Liu, A. P. S. Meliopoulos, R. Fan, and L. Sun, "Dynamic state estimation based protection of microgrid circuits," in Proc. IEEE Power \& Energy Society General Meeting (PESGM), 2015, pp. 1-5.
[29] S. Choi et al., "Effective real-time operation and protection scheme of microgrids using distributed dynamic state estimation," IEEE Transactions on Power Delivery, vol. 32, no. 1, pp. 504-514, 2016.

[30] X. Dong et al., "Traveling wave based single-phase-to-ground protection method for power distribution system," CSEE Journal of power and Energy Systems, vol. 1, no. 2, pp. 75-82, 2015.

[31] X. Li et al., "Traveling wave-based protection scheme for inverterdominated microgrid using mathematical morphology," IEEE Transactions on Smart Grid, vol. 5, no. 5, pp. 2211-2218, 2014.

[32] N. Davydova and G. Hug, "Traveling wave based protection for medium voltage grids with distributed generation," in Proc. IEEE Manchester PowerTech, 2017, pp. 1-6.

[33] N. Davydova and G. Hug, "Travelling wave protection with disturbance classification for distribution grids with distributed generation," The Journal of Engineering, vol. 2018, no. 15, pp. 830-835, 2018.

[34] H. Lin et al., "Adaptive protection combined with machine learning for microgrids," IET Generation, Transmission \& Distribution, vol. 13, no. 6, pp. 770-779, 2019.

[35] T. S. Abdelgayed et al., "Fault detection and classification based on co-training of semisupervised machine learning," IEEE Transactions on Industrial Electronics, vol. 65, no. 2, pp. 1595-1605, 2017.

[36] M. Mishra and P. K. Rout, "Detection and classification of micro-grid faults based on HHT and machine learning techniques," IET Generation, Transmission \& Distribution, vol. 12, no. 2, pp. 388-397, 2017.

[37] M. Manohar, E. Koley, and S. Ghosh, "Enhancing the reliability of protection scheme for PV integrated microgrid by discriminating between array faults and symmetrical line faults using sparse auto encoder," IET Renewable Power Generation, vol. 13, no. 2, pp. 308-317, 2018.

[38] Q. Cui and S. Li, "A microgrid protection scheme with conventional relay measurements," in Proc. IEEE Power \& Energy Society General Meeting (PESGM), 2018, pp. 1-5.

[39] M. Uzair, L. Li, and J. G. Zhu, "Identifying line-to-ground faulted phase in low and medium voltage AC microgrid using principal component analysis and supervised machine-learning," in Proc. Australasian Universities Power Engineering Conference (AUPEC), 2018, pp. 1-6.

[40] J. James, Y. Hou, A. Y. Lam, and V. O. Li, "Intelligent fault detection scheme for microgrids with wavelet-based deep neural networks," IEEE Transactions on Smart Grid, vol. 10, no. 2, pp. 1694-1703, 2017.

[41] D. P. Mishra, S. R. Samantaray, and G. Joos, "A combined wavelet and data-mining based intelligent protection scheme for microgrid," IEEE Transactions on Smart Grid, vol. 7, no. 5, pp. 2295-2304, 2015.

[42] M. Manohar et al., "A reliable fault detection and classification scheme based on wavelet transform and ensemble of SVM for microgrid protection," in Proc. 3rd Intl. Conf. on Applied and Theoretical Computing and Communication Technology (iCATccT), 2017, pp. 24-28.

[43] L. Jin et al., "Fault analysis of microgrid and adaptive distance protection based on complex wavelet transform," in Proc. International Power Electronics and Application Conf. and Exposition, 2014, pp. 360-364.

[44] T. S. Aghdam, H. K. Karegar, and H. H. Zeineldin, "Variable tripping time differential protection for microgrids considering DG stability," IEEE Transactions on Smart Grid, vol. 10, no. 3, pp. 2407-2415, 2018.

[45] B. Satuyeva et al., "Q-learning based protection scheme for microgrid using multi-agent system," in Proc. International Conference on Smart Energy Systems and Technologies (SEST), 2019, pp. 1-6.

[46] H. S. Samkari and B. K. Johnson, "Multi-agent protection scheme for resilient microgrid systems with aggregated electronically coupled distributed energy resources," in Proc. 44th Annual Conference of the IEEE Industrial Electronics Society (IECON), 2018, pp. 752-757.

[47] M. J. Daryani, A. E. Karkevandi, and O. Usta, "Multi-agent approach to wide-area integrated adaptive protection system of microgrid for preand post-contingency conditions," in Proc. IEEE PES Innovative Smart Grid Technologies Conference Europe, 2018, pp. 1-6.

[48] H. F. Habib et al., "Decentralized multi-agent system for protection and the power restoration process in microgrids," in Proc. 9th Annual IEEE Green Technologies Conference (GreenTech), 2017, pp. 358-364.

[49] H. F. Habib et al., "A multiagent system for simple overcurrent protection of microgrids with distributed generation," in Proc. IEEE Industry Applications Society Annual Meeting (IAS), 2018, pp. 1-8.

[50] S. Ranjbar et al., "Fault detection in microgrids using combined classification algorithms and feature selection methods," in Proc. Intl. Conf. on Protection and Automation of Power System (IPAPS), 2019, pp. 17-21.

[51] S. Kar, S. Samantaray, and M. D. Zadeh, "Data-mining model based intelligent differential microgrid protection scheme," IEEE Systems Journal, vol. 11, no. 2, pp. 1161-1169, 2015. 